\title{
EXTREMAL BINARY SELF-DUAL CODES OF LENGTHS 42 AND 44 WITH NEW WEIGHT ENUMERATORS
}

\author{
Daniel B. DALAN
}

(Received 14 November 2002 and revised 17 April 2003)

\begin{abstract}
A paper of Conway and Sloane (J. H. Conway and N. J. A. Sloane. A new upper bound on the minimal distance of self-dual codes. IEEE Trans. Inform. Theory 36 (1990), 1319-1333) gives the putative weight enumerators of extremal self-dual binary codes for lengths $n \leq 64$ and $n=72$. The construction of such codes with putative weight enumerators has been one of the fundamental problems in coding theory. In this paper, we construct extremal self-dual binary $[42,21,8]$ and extremal self-dual binary $[44,22,8]$ codes whose weight enumerators are not yet known to exist.
\end{abstract}

\section{Introduction}

We let $V$ be a vector space over the finite field of two elements. If $C \subseteq V$, then $C$ is called a binary linear code. An element of $C$ is called a codeword.

Let $u=\left(u_{1}, \ldots, u_{n}\right), v=\left(v_{1}, \ldots, v_{n}\right) \in V^{n}$. The standard inner product on $V^{n}$ is defined by

$$
u \cdot v=\sum_{j=1}^{n} u_{j} v_{j} \quad(\bmod 2) .
$$

We define the dual of $C$ by

$$
C^{\perp}=\left\{u \in V^{n} \mid u \cdot v=0 \forall v \in C\right\} .
$$

If $C \subset C^{\perp}$ (respectively $C=C^{\perp}$ ) then $C$ is called self-orthogonal (respectively self-dual). 
Two codes are said to be equivalent if one is obtained from the other by a permutation of coordinates. If $\Phi$ is a mapping that sends $C$ into itself, then $\Phi$ is called an automorphism of $C$ and the set of all automorphisms of $C$ forms a group under composition called the automorphism group of $C$, which is denoted by $\operatorname{Aut}(C)$ in this paper.

For $u \in V^{n}$, the weight of $u$ (denoted by wt $(u)$ ) is the number of non-zero coordinates of $u$. The minimum weight of a code $C$ is defined by

$$
d(C)=\min \{\operatorname{wt}(u) \mid u \in C, u \neq 0\} .
$$

A fundamental problem in coding theory is to find a code $C \subset V^{n}$ with $\operatorname{dim}$ $C=k$ such that $d(C)$ is as large as possible, for given positive integers $n$ and $k$.

A code $C$ is called doubly-even or Type II if for all $u \in C$,

$$
\mathrm{wt}(u) \equiv 0(\bmod 4)
$$

holds. A code which is not doubly-even is called singly-even or Type I code.

THEOREM 1. (Rains [13]) If C is a Type I code of length $n$, then

$$
d(C) \leq 4\left[\frac{n}{24}\right]+6 \quad \text { if } n \equiv 22(\bmod 24)
$$

or

$$
d(C) \leq 4\left[\frac{n}{24}\right]+4 \text { if } n \not \equiv 22(\bmod 24) .
$$

A self-dual code is called extremal if it has the highest minimum weight for that length.

Let $C$ be a code and let $A_{i}$ denote the number of codewords in $C$ of weight $i$. The weight enumerator of $C$ is a polynomial in $y$ (where $y$ is an indeterminate) expressed as

$$
W_{n}(y)=\sum_{i=1}^{n} A_{i} y^{i} .
$$

In [6], all the weight enumerators of extremal self-dual codes of lengths $n \leq 64$ and $n=72$ were computed. In particular, the weight enumerator of code $C$ of length 42 is either of the following:

$$
W_{42,1}(y)=1+(84+8 \beta) y^{8}+(1449-24 \beta) y^{10}+\cdots,
$$

or

$$
W_{42,2}(y)=1+164 y^{8}+15088 y^{10}+\cdots .
$$


For $W_{42,1}(y), \beta$ takes on the values $0 \leq \beta \leq 60$ and there exist self-dual codes with $\beta=0, \ldots, 16,18,20,22,24,26,32,42$ (see [3] and the references therein).

While for a code $C$ of length 44, the weight enumerator is either of the following:

$$
W_{44,1}(y)=1+(44+4 \beta) y^{8}+(976-8 \beta) y^{10}+\cdots,
$$

or

$$
W_{44,2}(y)=1+(44+4 \beta) y^{8}+(1232-8 \beta) y^{10}+\cdots .
$$

For $W_{44,1}(y), \beta$ takes on the values $10 \leq \beta \leq 122$ and there exist self-dual codes with $\beta=10, \ldots, 48,50,52,54,56,58,60,62,64,66,70,82,90,122$, while for $W_{44,2}(y), \beta$ takes on the values $0 \leq \beta \leq 154$ and there exist self-dual codes with $\beta=0, \ldots, 48,50,52, \ldots, 56,58,60,62,64,66,68,70,72,74,76,82,86,90$, $104,154$ (see $[\mathbf{3}, \mathbf{7 - 9}])$.

It is the purpose of this paper to fill in some of the gaps on the values of $\beta$ in $W_{42,1}(y), W_{44,1}(y)$ and $W_{44,2}(y)$.

\section{Construction of the new codes}

The vector in $V^{n}$ whose entries are all 1 is called the all one vector (denoted by $\mathbf{1}$ ) and every self-dual code contains 1 .

Self-dual codes $C_{1}, C_{2}$ of length $n$ are called neighbors to each other if $C_{1} \cap C_{2}$ has dimension $(n / 2-1)$. Note that this intersection necessarily contains $\mathbf{1}$. Since $C_{1}$ and $C_{2}$ are self-dual, $\operatorname{dim} C_{1}=\operatorname{dim} C_{2}$. It is known that neighbors are a way of constructing new self-dual codes from known self-dual codes and such results can be found in [1] and [6].

We solved our problem using a computer package called Magma and used a modified algorithm which was used in [11]. We will discuss what we did without writing down the Magma functions.

A self-dual code $C$ of length $n$ has $2^{n / 2-1}-1$ subcodes containing the all one vector $\mathbf{1}$. As a first step, we compute the automorphism group $A$ of $C$ and classify the neighbors of $C$ up to the action of $A$. To this end, it suffices to classify subcodes of codimension 1 of $C$ containing $\mathbf{1}$. Moreover, if we regard $C$ as a vector space over $V$, then the subcodes of codimension 1 of $C$ containing 1 are in one-to-one correspondence with the non-zero elements of the dual space of the quotient $M$ of $C$ by the one-dimensional space spanned by 1 .

This then leads us to construct the action of $A$ on $M$. To do this, we define a permutation module $P$ for $A$ over $V$ and construct a submodule $N$ of $P$ generated by 
the vectors in $C$. Now we define $M=N /\langle\mathbf{1}\rangle$. As $N$ is defined to be an $A$-module, $M$ is also an $A$-module.

Next, we construct the matrix representation $G$ of $A$ in the dual space of $M$ and define $X$ as the set of orbit representatives of $G$ on the set of non-zero vectors of $V^{n / 2-1}$.

Let $x$ be an element of $X$. As we wish to regard $x$ as an element of the dual space of $M$, we should be able to construct a subcode of codimension 1 of $M$. We will show how this can be done. First, we define $N_{0}$ as a subspace of the vector space of $P$ generated by the vectors in $C$. We let $M_{0}=N_{0} /\langle\mathbf{1}\rangle$. We also let $K_{1}$ be a vector space of degree 1 over $V$. Now we define the dual of $M_{0}$ to be the vector space of homomorphisms of $M_{0}$ and $K_{1}$. The subcode $M_{h}$ of codimension 1 of $M$ can be defined by the kernel of $x$ in the dual of $M_{0}$.

Now $M_{h}$ is a subcode of codimension 1 of $M$. We can construct a subcode of codimension 1 (say $H$ ) of $C$ by taking the preimage of the natural mapping from $C$ to its quotient by $\mathbf{1}$.

Finally, we can construct the neighbor of $C$ sharing $H$. We let $H_{0}$ be the vector space defined by $H$ and let $H_{\mathrm{d}}$ be vector space defined by the dual of $H$. We define $T$ to be the sequence of transversals for $H_{\mathrm{d}}$ in $H_{0}$ such that the weight of each transversal is equal to 8 . We then take the first element of $T$ (say $t$ ) and define a subspace $C^{\prime}$ of $H_{\mathrm{d}}$ generated by the elements of $H$ and $t$. Now, $C^{\prime}$ is the neighbor of $C$ sharing $H$. By doing this procedure to all the elements of $X$, we can construct all the neighbors of $C$.

We give some remarks on the algorithm mentioned above. The code $C$ has any of the following parameters: [42, 21, 6], [42, 21, 8], [44, 22, 6] or [44, 22, 8]. It is possible to find all codes with putative weight enumerators by iterating this algorithm but it is difficult to find an appropriate code $C$. We can choose $C$ to have $d(C)$ equal to two or four. However, the probability of constructing the neighbors of these codes that can attain a minimum distance eight is lower than if we choose $C$ to have $d(C)$ equal to six or eight. We mainly chose $C$ to have $d(C)=6$, since work in this area has never existed before.

\section{Results}

We ran the algorithm on the [42, 21,8] and [44, 22, 8] codes found in [14]. We remark that even the results were recursively used thus making our work an exhaustive search. The generator matrix for the $[42,21,8]$ code found in [14] is 
defined as

$$
\left(\begin{array}{cccccc}
1 \ldots 1 & 0 \ldots 0 & 0 \ldots 0 & 1 \ldots 1 & 0 \ldots 0 & 0 \ldots 0 \\
0 \ldots 0 & 1 \ldots 1 & 0 \ldots 0 & 0 \ldots 0 & 1 \ldots 1 & 0 \ldots 0 \\
0 \ldots 0 & 0 \ldots 0 & 1 \ldots 1 & 0 \ldots 0 & 0 \ldots 0 & 1 \ldots 1 \\
S & S & S & 0 \ldots 0 & 0 \ldots 0 & 0 \ldots 0 \\
T & T & 0 \ldots 0 & 0 \ldots 0 & 0 \ldots 0 & 0 \ldots 0 \\
T & 0 \ldots 0 & T & 0 \ldots 0 & 0 \ldots 0 & 0 \ldots 0 \\
0 \ldots 0 & 0 \ldots 0 & 0 \ldots 0 & S & S & S \\
0 \ldots 0 & 0 \ldots 0 & 0 \ldots 0 & T & T & 0 \ldots 0 \\
0 \ldots 0 & 0 \ldots 0 & 0 \ldots 0 & T & 0 \ldots 0 & T
\end{array}\right)
$$

where $S, T$ are $(3 \times 7)$ circulant matrices with first rows 1110100, 1001011, respectively, and the generator matrix for the [44, 22, 8] code found in [14] is defined as

$$
\left(\begin{array}{cccc}
1 \ldots 1 & 0 \ldots 0 & 1 \ldots 1 & 0 \ldots 0 \\
0 \ldots 0 & 1 \ldots 1 & 0 \ldots 0 & 1 \ldots 1 \\
M & N & 0 \ldots 0 & 0 \ldots 0 \\
0 \ldots 0 & 0 \ldots 0 & M & N
\end{array}\right)
$$

where $M, N$ are $(10 \times 11)$ circulant matrices with first rows $01 \ldots 1,11011100010$, respectively.

$C_{42,1}$ is a $[42,21,6]$ code and is a neighbor of the [42, 21, 8] code found in [14]. $C_{42,2}$ is a neighbor of $C_{42,1}$ and is a $[42,21,8]$ code. The automorphism groups of these codes are permutation groups acting on a set of cardinality 42 . Their orders and generators are presented in Table 1.

$C_{44, j}(j=1, \ldots, 9)$ are $[44,21,6]$ codes and all of which are neighbors of the $[44,22,8]$ code found in [14]. The automorphism groups of these codes are permutation groups acting on a set of cardinality 44. Their orders and generators are presented in Table 2.

A neighbor of $C$ can be constructed from one vector $v \in C$ with the subcode of $C$ consisting of codewords orthogonal to $v$. Thus, giving one vector is enough since the subcode is automatically obtained from the vector. We present the vectors that define the generator matrices of the codes $C_{42,1}, C_{42,2}$ and $C_{44, j}(j=1, \ldots, 9)$ in hexadecimal notation (i.e. $\mathbf{0}=(0,0,0,0), \mathbf{1}=(0,0,0,1), \ldots, \mathbf{F}=(1,1,1,1))$ and note that they are right justified. The vectors are given in Tables 3 and 4 .

By using the code $C_{42,1}$ and the defining vector [2, 0, 0, 0, 0, 0, A, 4, 2, A, 1], we found the code $C_{1}^{\prime}$ with the weight enumerator $W_{42,1}$ for a new value $\beta=17$. Similarly, we found the codes $C_{i}^{\prime}(i=2,3,4)$ for $\beta=19,21,28$ from $C_{42,1}, C_{1}^{\prime}$, 

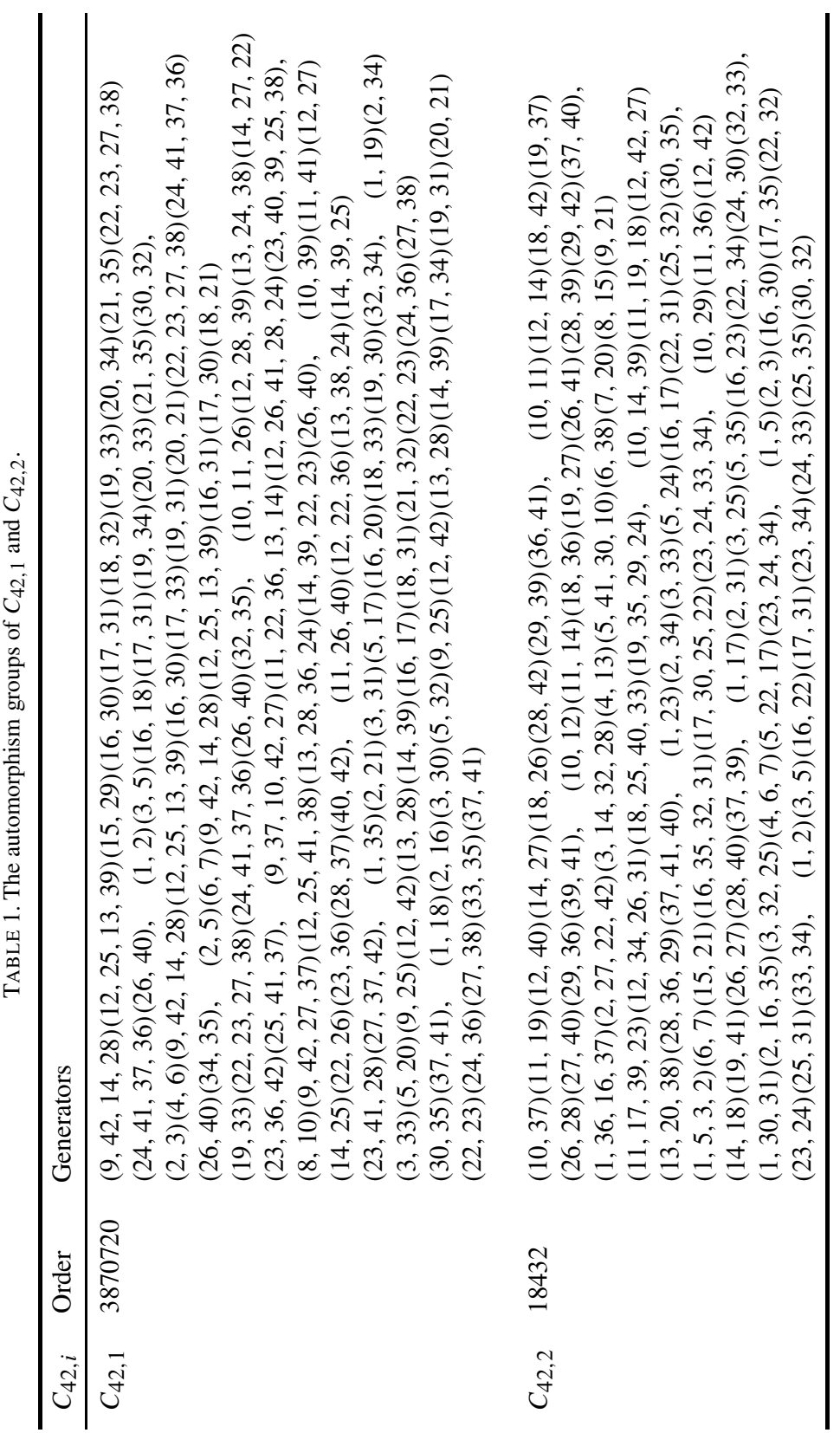


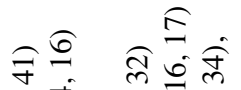

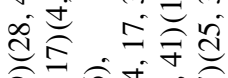
तेलंब्तें ते

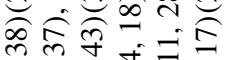

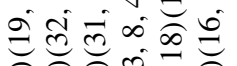

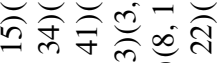

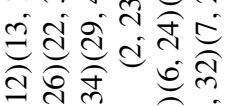

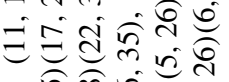
तิ

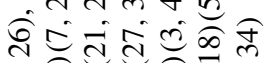

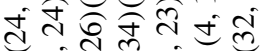

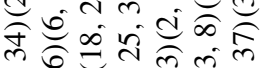

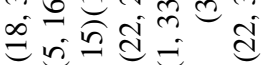

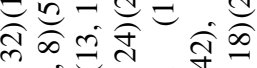

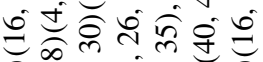

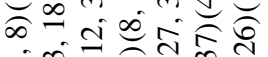

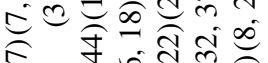
音 0 त อิ केष्वेत्रत nิ) 公的矛过

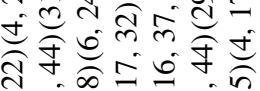
लं क्र

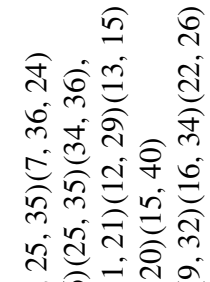

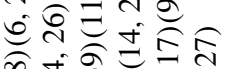
过

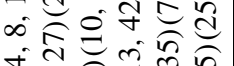
+ ते m.

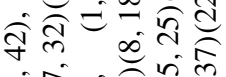
过它命的 过命 ซे ले लु 娄告

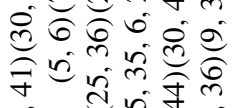
过它

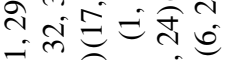
ป્ปิ่

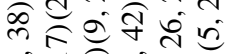
包完过

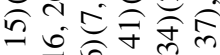
m তิ่

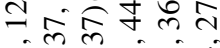

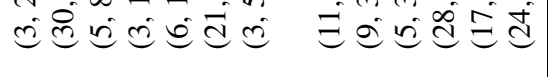

$\stackrel{\infty}{\sim}$

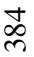

Ũ 


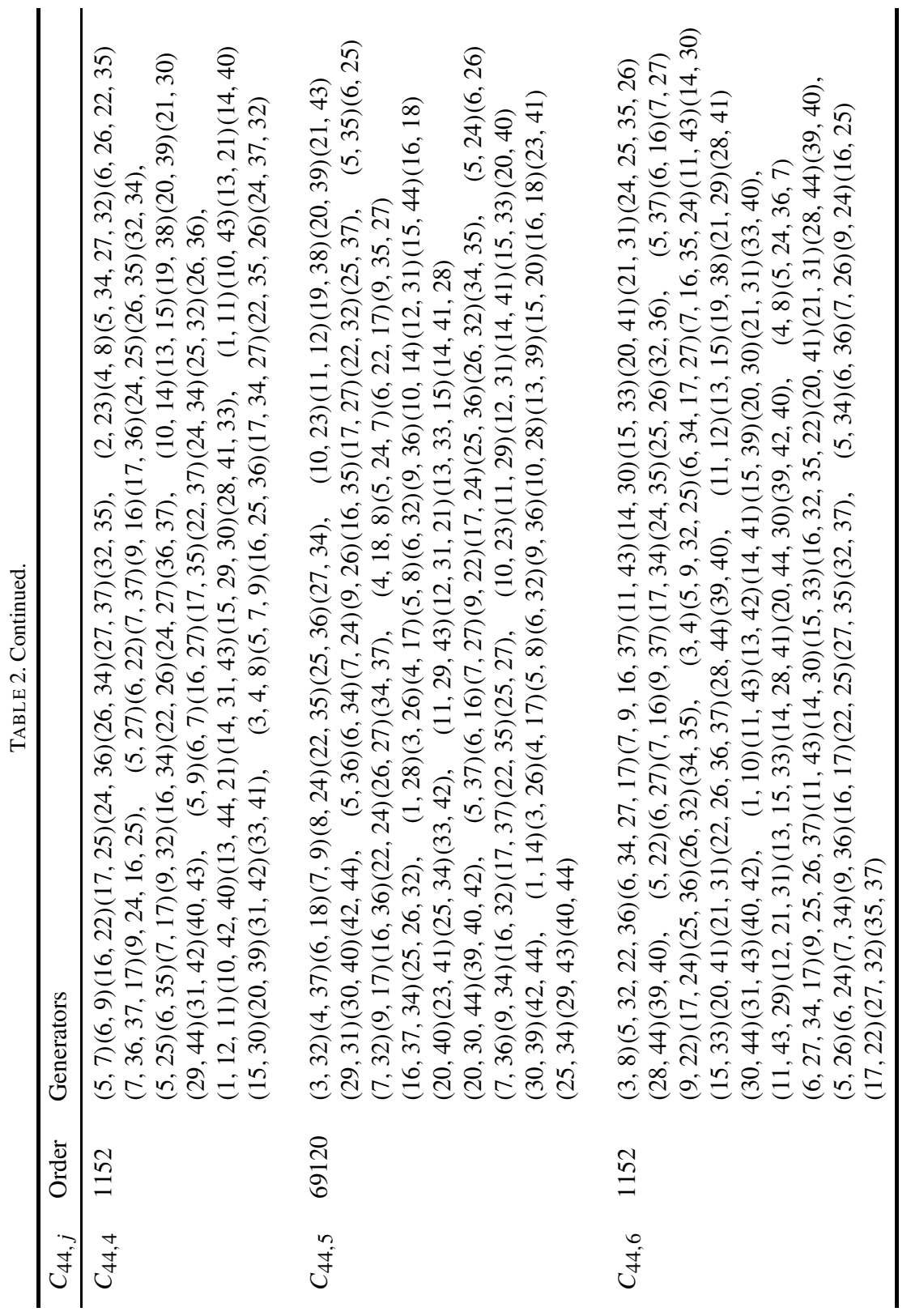




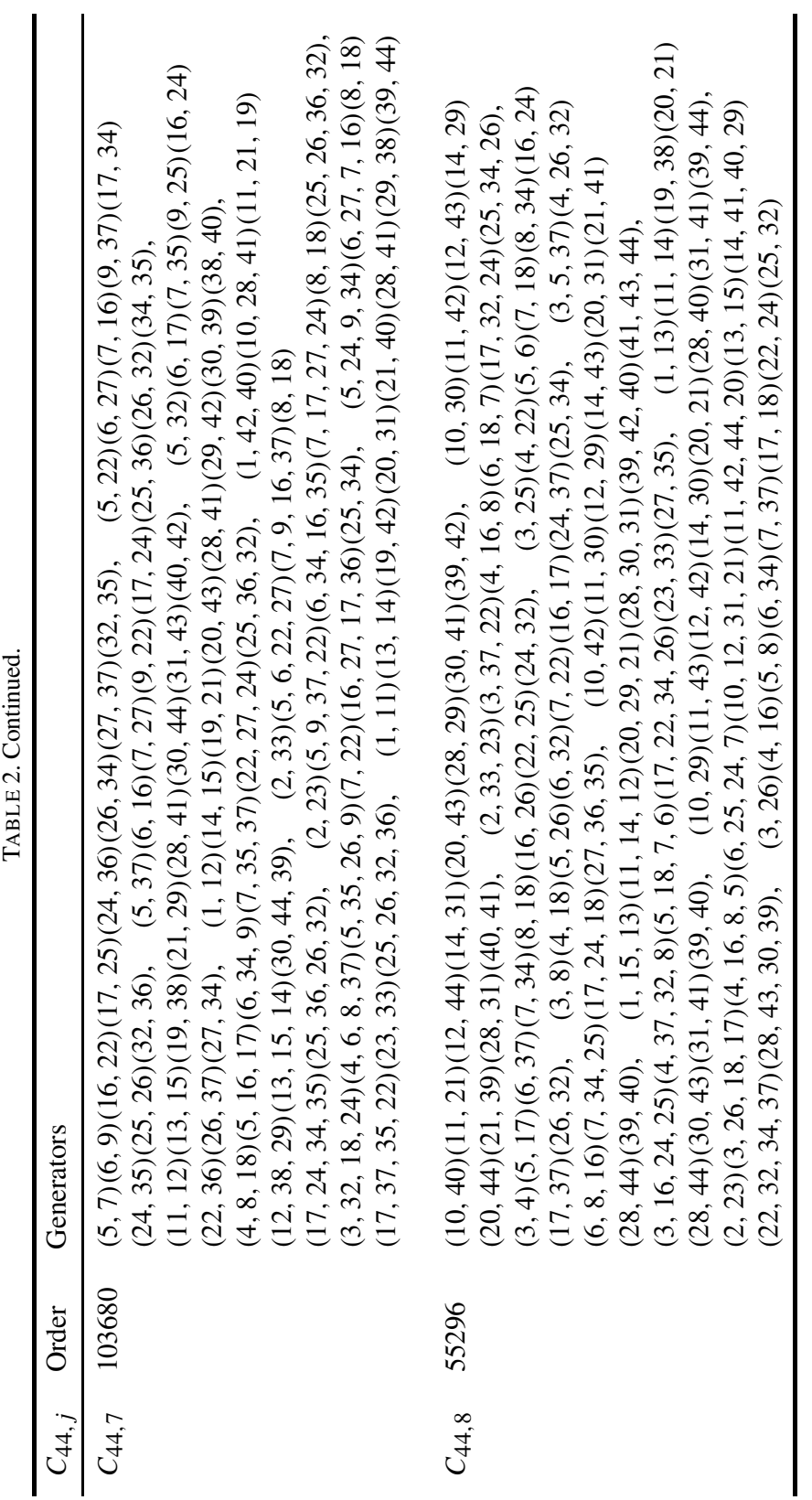




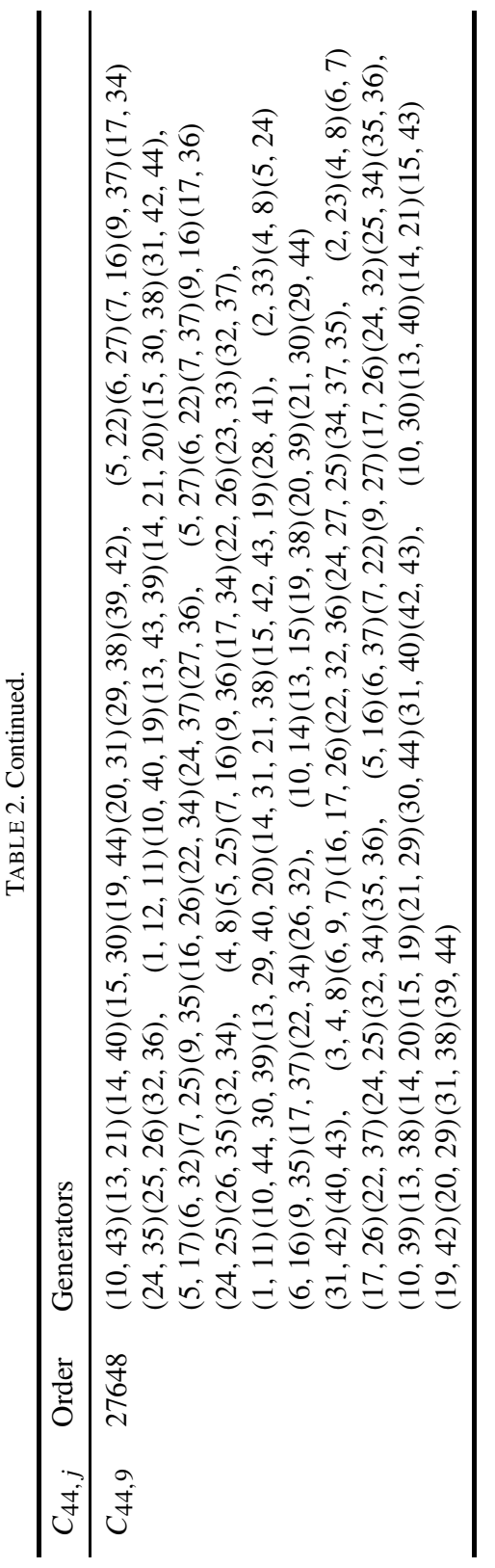


TABLE 3 . The vectors that define $C_{42,1}$ and $C_{42,2}$.

\begin{tabular}{ccl}
\hline$C_{42, i}$ & $d\left(C_{42, i}\right)$ & Defining vector \\
\hline$C_{42,1}$ & 6 & {$[2,0,0,0,0,1,1,9, A, 6,4]$} \\
$C_{42,2}$ & 8 & {$[2,0,0,0,0,0, D, 1,9,2,6]$} \\
\hline
\end{tabular}

TABLE 4 . The vectors that define $C_{44, j}(j=1, \ldots, 9)$.

\begin{tabular}{lcl}
\hline$C_{44, j}$ & $d\left(C_{44, j}\right)$ & Defining vector \\
\hline$C_{44,1}$ & 6 & {$[8,0,0,0,0,2,0,0, E, C, B]$} \\
$C_{44,2}$ & & {$[8,0,0,0,0,3,9,2,5, A, A]$} \\
$C_{44,3}$ & & {$[8,0,0,0,0,2, D, F, 1,5,3]$} \\
$C_{44,4}$ & & {$[8,0,0,0,0,2,0,0,8,7, F]$} \\
$C_{44,5}$ & {$[8,0,0,0,0,3,4, C, D, 3,2]$} \\
$C_{44,6}$ & {$[8,0,0,0,0,3, B, C, 6, C, 5]$} \\
$C_{44,7}$ & {$[8,0,0,0,0,3, A, E, 6,9,0]$} \\
$C_{44,8}$ & {$[8,0,0,0,0,2, E, 8, B, E, C]$} \\
$C_{44,9}$ & {$[8,0,0,0,0,2,3,0,3,5,9]$} \\
\hline
\end{tabular}

TABLE 5. New extremal binary codes with weight enumerator $W_{42,1}(y)$.

\begin{tabular}{lclc}
\hline$C^{\prime}$ & $\beta$ & Defining vector & Neighbor of \\
\hline$C_{1}^{\prime}$ & 17 & {$[2,0,0,0,0,0, A, 4,2, A, 1]$} & $C_{42,1}$ \\
$C_{2}^{\prime}$ & 19 & {$[2,0,0,0,0,0, D, A, 3,1,7]$} & $C_{42,1}$ \\
$C_{3}^{\prime}$ & 21 & {$[2,0,0,0,0,0, A, 4,2, A, 1]$} & $C_{1}^{\prime}$ \\
$C_{4}^{\prime}$ & 28 & {$[2,0,0,0,0,1,6, D, F, C, D]$} & $C_{42,2}$ \\
\hline
\end{tabular}

$C_{42,2}$, respectively. Examples of $C_{i}^{\prime}(i=1, \ldots, 4)$ are listed in Table 5. Thus we have the following.

PROpOSITION 1. There exist extremal self-dual $[42,21,8]$ codes with weight enumerator $W_{42,1}(y)$ for $\beta=17,19,21,28$.

By using $C_{44,1}$ and the defining vector [8, 0, 0, 0, 0, 2, E, F, 0, A, 4], we found the code $C_{1}^{\prime \prime}$ with the weight enumerator $W_{44,1}$ for a new value $\beta=49$. Similarly, we 


\begin{tabular}{cclc}
\multicolumn{6}{c}{ TABLE 6 . New extremal binary codes with weight enumerator $W_{44,1}(y)$} \\
\hline$C^{\prime \prime}$ & $\beta$ & Defining vector & Neighbor of \\
\hline$C_{1}^{\prime \prime}$ & 49 & {$[8,0,0,0,0,2, E, F, 0, A, 4]$} & $C_{44,1}$ \\
$C_{2}^{\prime \prime}$ & 51 & {$[8,0,0,0,0,3,9,2,5, A, A]$} & $C_{44,2}$ \\
$C_{3}^{\prime \prime}$ & 53 & {$[8,0,0,0,0,3,5,2,3, A, A]$} & $C_{44,3}$ \\
$C_{4}^{\prime \prime}$ & 55 & {$[8,0,0,0,0,2,4,3,2,6,9]$} & $C_{44,4}$ \\
$C_{5}^{\prime \prime}$ & 57 & {$[8,0,0,0,0,3, B, 6,8,4,0]$} & $C_{44,5}$ \\
$C_{6}^{\prime \prime}$ & 59 & {$[8,0,0,0,0,2, E, E, 5,2,4]$} & $C_{44,1}$ \\
$C_{7}^{\prime \prime}$ & 63 & {$[8,0,0,0,0,3,4, C, D, 3,2]$} & $C_{44,6}$ \\
$C_{8}^{\prime \prime}$ & 65 & {$[8,0,0,0,0,2, E, 2, B, E, 9]$} & $C_{11}^{\prime \prime}$ \\
$C_{9}^{\prime \prime}$ & 67 & {$[8,0,0,0,0,3,3,8,4,9, B]$} & $C_{44,7}$ \\
$C_{10}^{\prime \prime}$ & 68 & {$[8,0,0,0,0,2,1,8,6, A, F]$} & $C_{5}^{\prime \prime}$ \\
$C_{11}^{\prime \prime}$ & 72 & {$[8,0,0,0,0,2, E, 2, B, E, 9]$} & $C_{44,8}$ \\
$C_{12}^{\prime \prime}$ & 86 & {$[8,0,0,0,0,2, E, 8, B, E, C]$} & $C_{44,9}$ \\
\hline
\end{tabular}

TABLE 7. New extremal binary codes with weight enumerator $W_{44,2}(y)$.

\begin{tabular}{lclc}
\hline$C^{\prime \prime \prime}$ & $\beta$ & Defining vector & Neighbor of \\
\hline$C_{1}^{\prime \prime \prime}$ & 49 & {$[8,0,0,0,0,3, A, 4, D, 2, C]$} & $C_{44,9}$ \\
$C_{2}^{\prime \prime \prime}$ & 51 & {$[8,0,0,0,0,3,5, E, 5,2,1]$} & $C_{44,9}$ \\
$C_{3}^{\prime \prime \prime}$ & 59 & {$[8,0,0,0,0,2,1,8,6, E, 4]$} & $C_{44,7}$ \\
\hline
\end{tabular}

found the codes $C_{i}^{\prime \prime}(i=2, \ldots, 12)$ for $\beta=51,53,55,57,59,63,65,67,68,72,86$ from $C_{44,2}, C_{44,3}, C_{44,4}, C_{44,5}, C_{44,1}, C_{44,6}, C_{11}^{\prime \prime}, C_{44,7}, C_{5}^{\prime \prime}, C_{44,8}, C_{44,9}$, respectively. Examples of $C_{i}^{\prime \prime}(i=1, \ldots, 12)$ are listed in Table 6 . Thus we have the following.

Proposition 2. There exist extremal self-dual $[44,22,8]$ codes with weight enumerator $W_{44,1}(y)$ for $\beta=49,51,53,55,57,59,63,65,67,68,72,86$.

By using the code $C_{44,9}$ and the defining vector [8, 0, 0, 0, 0, 3, A, 4, D, 2, C], we found the code $C_{1}^{\prime \prime \prime}$ with the weight enumerator $W_{44,2}$ for a new value $\beta=49$. Similarly we found the codes $C_{2}^{\prime \prime \prime}, C_{3}^{\prime \prime \prime}$ from $C_{44,9}, C_{44,7}$, respectively. Examples of $C_{i}^{\prime \prime \prime}(i=1,2,3)$ are listed in Table 7 . Thus we have the following. 
Proposition 3. There exist extremal self-dual [44,22,8] codes with weight enumerator $W_{44,2}(y)$ for $\beta=49,51,59$.

\title{
REFERENCES
}

[1] R. A. Brualdi and V. S. Pless. Weight enumerators of self-dual codes. IEEE Trans. Inform. Theory 37 (1991), 1222-1225.

[2] I. Boukliev and S. Buyuklieva. Some new extremal self-dual codes with lengths 44, 50, 54, and 58. IEEE Trans. Inform. Theory 44 (1998), 809-812.

[3] S. Bouyuklieva. A method for constructing self-dual codes with an automorphism of order 2. IEEE Trans. Inform. Theory 46 (2000), 496-504.

[4] S. Buyuklieva. New extremal self-dual codes of lengths 42 and 44. IEEE Trans. Inform. Theory 43 (1997), 1607-1612.

[5] S. Buyuklieva. On the binary self-dual codes with an automorphism of order 2. Des. Codes Cryptogr. 12 (1997), 39-48.

[6] J. H. Conway and N. J. A. Sloane. A new upper bound on the minimal distance of self-dual codes. IEEE Trans. Inform. Theory 36 (1990), 1319-1333.

[7] D. B. Dalan. New extremal type I codes of lengths 40,42, and 44. Des. Codes Cryptogr. To appear.

[8] D. B. Dalan. New extremal binary [44, 22, 8] codes. IEEE Trans. Inform. Theory 49 (2003), 747748 .

[9] S. T. Dougherty, T. A. Gulliver and M. Harada. Extremal binary self-dual codes. IEEE Trans. Inform. Theory 43 (1997), 2036-2047.

[10] F. J. Macwilliams and N. J. A. Sloane. The Theory of Error-correcting Codes. North-Holland, New York, 1977.

[11] A. Munemasa. On the enumeration of binary self-dual codes. Preprint.

[12] V. Pless. Coding constructions. Handbook of Coding Theory. Eds V. S. Pless and W. C. Huffman. Elsevier, Amsterdam, 1998.

[13] E. Rains. Shadow bounds for self-dual codes. IEEE Trans. Inform. Theory 44 (1998) 134-139.

[14] R. Ruseva and V. Y. Yorgov. Two extremal codes of length 42 and 44. Probl. Pered. Inform. 29 (1993) 99-103. In Russian.

[15] E. Rains and N. J. A Sloane. Self-dual codes. Handbook of Coding Theory. Eds V. S. Pless and W. C. Huffman. Elsevier, Amsterdam, 1998.

[16] V. Yorgov. New extremal singly-even self-dual codes of length 44. Proc. 6th Joint Swedish-Russian Int. Workshop on Information Theory, Mölle, Sweden, 1993, pp. 372-375.

\author{
Daniel B. Dalan \\ Graduate School of Mathematics 33 \\ Kyushu University \\ Fukuoka 812-8581 \\ Japan \\ (E-mail: sporadic235@hotmail.com)
}

